\title{
Analyzing consensus approaches in fuzzy group decision making: advantages and drawbacks
}

\author{
F.J. Cabrerizo • J.M. Moreno · I.J. Pérez • E. Herrera-Viedma
}

Received: date / Accepted: date

\begin{abstract}
Two processes are necessary to solve group decision making problems: a consensus process and a selection process. The consensus process is necessary to obtain a final solution with a certain level of agreement between the experts while the selection process is necessary to obtain such a final solution. Clearly, it is preferable that the set of experts reach a high degree of consensus before applying the selection process. In order to measure the degree of consensus, different approaches have been proposed. For example, we can use hard consensus measures, which vary between 0 (no consensus or partial consensus) and 1 (full consensus), or soft consensus measures, which assess the consensus degree in a more flexible way. The aim of this paper is to analyze the different consensus approaches in fuzzy group decision making problems and discuss their advantages and drawbacks. Additionally, we study the future trends.
\end{abstract}

Keywords Group decision making · Consensus process $\cdot$ Soft consensus measures $\cdot$ Future trends

\section{F.J. Cabrerizo}

Department of Software Engineering and Computer Systems,

Distance Learning University of Spain, 28040 Madrid, Spain

E-mail: cabrerizo@issi.uned.es

\section{J.M. Moreno}

Department of Information and Communication Engineering,

University of Murcia, 30100 Murcia, Spain

E-mail: jmmoreno@um.es

I.J. Pérez · E. Herrera-Viedma

Department of Computer Science and Artificial Intelligence,

University of Granada, 18071 Granada, Spain

E-mail: ijperez@decsai.ugr.es

E. Herrera-Viedma

E-mail: viedma@decsai.ugr.es

\section{Introduction}

In a classical Group Decision Making (GDM) situation there is a problem to solve, a solution set of possible alternatives, $X=\left\{x_{1}, \ldots, x_{n}\right\}$, and a group of two or more experts, $E=\left\{e_{1}, \ldots, e_{m}\right\}$, characterized by their own ideas, attitudes, motivations and knowledge, who express their opinions about this set of alternatives to achieve a common solution $[30,32,33]$. To do this, experts have to express their preferences by means of a set of evaluations over the set of alternatives.

GDM problems arise from many real-world situations [8]. To solve these problems, the experts are faced by applying two processes before obtaining a final solution $[20,28,29]$ : consensus process and selection process (see Fig. 1). The former consists in how to obtain the maximum degree of consensus or agreement between the set of experts on the solution set of alternatives. Normally, the consensus process is guided by a human figure called moderator $[11,28]$ who is a person that does not participate in the discussion but knows the agreement in each moment of the consensus process and is in charge of supervising and addressing the consensus process toward success, i.e., to achieve the maximum possible agreement and to reduce the number of experts outside of the consensus in each new consensus round. The latter refers to how to obtain the solution set of alternatives from the opinions on the alternatives given by the experts. It involves two different steps [15, 34]: aggregation of individual opinions and exploitation of the collective opinion. Clearly, it is preferable that the set of experts achieves a great agreement among their opinions before applying the selection process.

A consensus process is defined as a dynamic and iterative group discussion process, coordinated by a moderator helping experts bring their opinions closer. At 


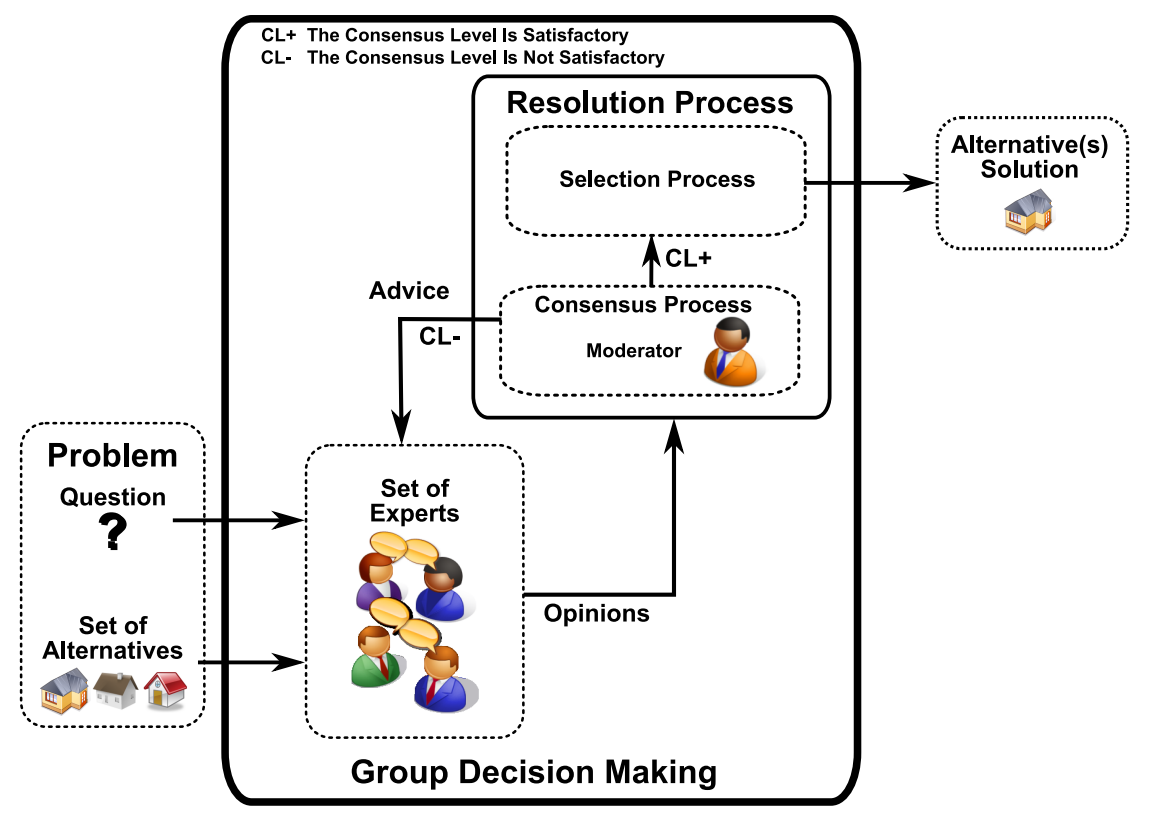

Fig. 1 Resolution process of a GDM problem

the beginning of every GDM problem, the set of experts has diverging opinions, then the consensus process is applied and, in each step, the degree of existing consensus among experts' opinions is measured. If the consensus degree is lower than a specified threshold, the moderator would urge experts to discuss their opinions further in an effort to bring them closer. Otherwise, the moderator would apply the selection process in order to obtain the final consensus solution to the GDM problem.

A natural question in the consensus process is how to measure the closeness among experts' opinions in order to obtain the consensus level. To do so, different approaches have been proposed. For instance, several authors have introduced hard consensus measures varying between 0 (no consensus or partial consensus) and 1 (full consensus) $[3,4,35,36]$. In this way, using hard consensus measures, a distance from consensus as a difference between some average preference matrix and one of several possible consensus preference matrices is determined in $[3,4]$. In [35] some measures of attitudinal similarity between individuals that is an extension of the classical Tanimoto coefficient are derived. Finally, a consensus measure based on $a$-cuts of the respective individual fuzzy preference matrices is derived in [36]. However, consensus as a full and unanimous agreement is far from being achieved in real situations and, even if it is, in such a situation, the consensus reaching process could be unacceptably costly. A more realistic approach is to use soft consensus measures [25-27], which assess the consensus degree in a more flexible way and, there- fore, reflect the large spectrum of possible partial agreements and guide the consensus process until widespread agreement (not always full) is achieved among experts. Soft consensus measures are based on the concept of coincidence [13], measured by means of similarity criteria defined among experts' opinions.

The aim of this paper is to analyze consensus approaches in fuzzy GDM problems to compute soft consensus measures and discuss their advantages and drawbacks. We identify three different coincidence criteria to compute soft consensus measures: (1) strict coincidence among preferences, (2) soft coincidence among preferences and (3) coincidence among solutions. Using these coincidence criteria two advanced consensus approaches have been proposed:

- Approaches allowing to generate recommendations to help experts change their opinions in order to obtain the highest degree of consensus possible [1921 ], and

- approaches adapting the consensus process to increase the agreement and to reduce the number of experts' preferences that should be changed after each consensus round [31].

The rest of the paper is organized as follows. In Section 2, we analyze the different approaches to obtain soft consensus measures in fuzzy GDM problems and illustrate an example of application. In Section 3, we discuss their advantages and drawbacks. The advanced consensus approaches are shown in Section 4. Finally, some concluding remarks are pointed out in Section 5. 


\section{Approaches to obtain soft consensus measures in fuzzy GDM problems}

As aforementioned, soft consensus measures are based on the coincidence concept [13], and we can identify three different consensus approaches to compute them: (1) consensus models based on strict coincidence among preferences, (2) consensus models based on soft coincidence among preferences, and (3) consensus models based on coincidence among on solutions. We describe them in more detail in the following subsections.

2.1 Consensus models based on strict coincidence among preferences

In this case, similarity criteria among preferences are used to compute the coincidence concept. It is assumed only two possible results: the total coincidence (value 1) or null coincidence (value 0). Some examples of this approach are the following:

- In [25], assuming fuzzy preference relations to represent experts' preferences, the first consensus model based on strict coincidence was defined. Given a particular alternative pair and two experts, if their preferences are equal, then they are in agreement (value 1), and, otherwise, they are in disagreement (value 0 ). Then, consensus measures are calculated across the global set of the alternatives in a hierarchical pooling process from the coincidence measured on experts' preferences and using the fuzzy majority concept represented by a linguistic quantifier [39].

- In [11,14], different consensus measures based on strict coincidence were presented assuming that experts' preferences are provided by means of linguistic preference relations. Applying the strict coincidence on preferences provided by the experts for each alternative pair, the expert group is divided into subsets, one subset for each possible linguistic label used to quality the preference on the alternative pair. Then, using the cardinalities of the subsets of experts three kinds of consensus measures are defined, each one associated to the three different levels of representation of a preference relation: alternative pair, individual alternative and global relation.

Assume a fuzzy GDM problem based on linguistic preference relations as in $[11,14]$, i.e., a GDM problem where the experts $E=\left\{e_{1}, \ldots, e_{m}\right\}$ express their preferences relations $P=\left\{P^{1}, \ldots, P^{m}\right\}$ on the set of alternatives $X$ using a linguistic term set $S=\left\{s_{0}, \ldots, s_{g}\right\}$ whose cardinality or granularity $\# S=g+1$, being $p_{i k}^{h} \in S$ the preference degree of alternative $x_{i}$ over alternative $x_{k}$ for the expert $e_{h}$. Additionally, the following properties are assumed $[18,22]$ :

1. The set $S$ is ordered: $s_{i} \geq s_{j}$ if $i \geq j$.

2. Negation operator: $\operatorname{Neg}\left(s_{i}\right)=s_{j}$ such that $j=g-i$.

3. Min operator: $\operatorname{Min}\left(s_{i}, s_{j}\right)=s_{i}$ if $s_{i} \leq s_{j}$.

4. Max operator: $\operatorname{Max}\left(s_{i}, s_{j}\right)=s_{i}$ if $s_{i} \geq s_{j}$.

Then, a consensus model based on strict coincidence could be carried out in the following steps:

1. Firstly, for each pair of experts $\left(e_{h}, e_{l}\right)(h=1, \ldots, m-$ $1, l=h+1, \ldots, m)$, a strict similarity matrix $S M^{h l}=$ $\left[s m_{i k}^{h l}\right], i, k=1, \ldots, n$, is obtained as follows:

$s m_{i k}^{h l}=\left\{\begin{array}{l}1, \text { if } p_{i k}^{h}=p_{i k}^{l} \\ 0, \text { otherwise }\end{array}\right.$

2. Then, a collective similarity matrix, $S M=\left[s m_{i k}\right]$, is obtained by aggregating all the similarity matrices using the arithmetic mean $\phi$ as the aggregation function:

$s m_{i k}=\phi\left(s m_{i k}^{h l}, h=1, \ldots, m-1, l=h+1, \ldots, m\right)$

Note 1: In this case, we have used the arithmetic mean as aggregation function $\phi$, although, different aggregation operators could be used according to the particular properties that we want to implement.

3. Computing the consensus degrees and proximity measures as in [11]:

(a) Consensus Degrees: Once the similarity matrices are computed, the consensus degrees are calculated as follows:

i. Level 1. Consensus degree on pairs of alternatives. The consensus degree, $\operatorname{cop}_{i k}$, on a pair of alternatives, $\left(x_{i}, x_{k}\right)$, is defined to measure the consensus degree among all the experts on that pair of alternatives. In this case, this is expressed by the element of the collective similarity matrix $S M$ :

$\operatorname{cop}_{i k}=s m_{i k}$

The closer $c o p_{i k}$ to 1 , the greater the agreement among all the experts on the pair of alternatives $\left(x_{i}, x_{k}\right)$. This measure will allow the identification of those pairs of alternatives with a poor level of consensus.

ii. Level 2. Consensus degree on alternatives. The consensus degree on the alternative $x_{i}$, called $c a_{i}$, is defined to measure the consensus degree among all the experts on that alternative:

$c a_{i}=\frac{\sum_{k=1 ; k \neq i}^{n}\left(\operatorname{cop}_{i k}+\operatorname{cop}_{k i}\right)}{2 n-2}$ 
These values can be used to propose the modification of preferences associated to those alternatives with a consensus degree lower than a minimal consensus threshold $\gamma$.

iii. Level 3. Consensus degree on the relation. The consensus degree on the relation, called $C R$, is defined to measure the global consensus degree among all the experts' opinions. It is computed as the average of all the consensus degrees on the alternatives:

$$
C R=\frac{\sum_{i=1}^{n} c a_{i}}{n}
$$

This is the value used to control the consensus situation.

Note 2: In [11] three kinds of consensus are proposed because they allow us to know the current state of consensus from different viewpoints and, therefore, to guide more correctly the consensus reaching process.

(b) Proximity Measures: To compute the proximity measures for each expert, we need to obtain the collective preference relation, $P^{c}=\left[p_{i k}^{c}\right]$, which summarizes preferences given by all the experts and is calculated by means of the aggregation of the set of individual preference relations $\left\{P^{1}, \ldots, P^{m}\right\}$ as follows:

$p_{i k}^{c}=\phi\left(p_{i k}^{1}, \ldots, p_{i k}^{m}\right)$

To do so, the Linguistic Ordered Weighted Averaging (LOWA) operator [12] can be used. The LOWA operator is based on the ordered weighted averaging (OWA) operator defined in [37], and on the convex combination of linguistic labels defined in [9]. In [12] it was shown that it is a rational operator to aggregate linguistic information that satisfies some important properties as commutativity, monotony, unanimity and neutrality.

Definition 1 Let $A=\left\{a_{1}, \ldots, a_{m}\right\}$ be a set of labels to be aggregated, then the LOWA operator, $\phi$, is defined as:

$\phi\left(a_{1}, \ldots, a_{m}\right)=W \cdot B^{T}=$

$\mathcal{C}^{m}\left\{w_{k}, b_{k}, k=1, \ldots, m\right\}=$

$w_{1} \odot b_{1} \oplus\left(1-w_{1}\right) \odot \mathcal{C}^{m-1}\left\{\beta_{h}, b_{h}, h=2, \ldots, m\right\}$

where $W=\left[w_{1}, \ldots, w_{m}\right]$, is a weighting vector, such that, $w_{i} \in[0,1]$ and $\Sigma_{i} w_{i}=1 . \beta_{h}=$ $w_{h} / \Sigma_{2}^{m} w_{k}, h=2, \ldots, m$, and $B=\left\{b_{1}, \ldots, b_{m}\right\}$ is a vector associated to $A$, such that, $B=\sigma(A)=$ $\left\{a_{\sigma(1)}, \ldots, a_{\sigma(m)}\right\}$, where, $a_{\sigma(j)} \leq a_{\sigma(i)} \forall i \leq j$, with $\sigma$ being a permutation over the set of labels $A . \mathcal{C}^{m}$ is the convex combination operator of $m$ labels and if $m=2$, then it is defined as $\mathcal{C}^{2}\left\{w_{i}, b_{i}, i=1,2\right\}=w_{1} \odot s_{j} \oplus\left(1-w_{1}\right) \odot s_{i}=s_{k}$, such that, $k=\min \left\{\mathcal{T}, i+\operatorname{round}\left(w_{1} \cdot(j-\right.\right.$ $i))\} s_{j}, s_{i} \in S,(j \geq i)$, where "round" is the usual round operation, and $b_{1}=s_{j}, b_{2}=s_{i}$. If $w_{j}=1$ and $w_{i}=0$ with $i \neq j \forall i$, then the convex combination is defined as: $\mathcal{C}^{m}\left\{w_{i}, b_{i}, i=\right.$ $1, \ldots, m\}=b_{j}$.

Using $P^{c}$, for each expert, $e_{h}$, a proximity matrix, $P M^{h}=\left[p m_{i k}^{h}\right]$, is obtained:

$p m_{i k}^{h}=\left\{\begin{array}{l}1, \text { if } p_{i k}^{h}=p_{i k}^{c} \\ 0, \text { otherwise }\end{array}\right.$

Finally, the computation of the proximity measures is carried out at three different level as follows:

i. Level 1. Proximity measure on pairs of alternatives. The proximity measure of an expert $e_{h}$ on a pair of alternatives $\left(x_{i}, x_{k}\right)$ to the group's one, called $p p_{i k}^{h}$, is expressed by the element $(i, k)$ of the proximity matrix $P M^{h}$ :

$$
p p_{i k}^{h}=p m_{i k}^{h}
$$

ii. Level 2. Proximity measure on alternatives. The proximity measure of an expert $e_{h}$ on an alternative $x_{i}$ to the group's one, called $p a_{i}^{h}$, is calculated as follows:

$p a_{i}^{h}=\frac{\sum_{k=1, k \neq i}^{n}\left(p p_{i k}^{h}+p p_{k i}^{h}\right)}{2 n-2}$

iii. Level 3. Proximity measure on the relation. The proximity measure of an expert $e_{h}$ on his/her preference relation to the group's one, called $p r^{h}$, is calculated as the average of all proximity measures on the alternatives:

$$
p r^{h}=\frac{\sum_{i=1}^{n} p a_{i}^{h}}{n}
$$

Given an expert, if his or her proximity measure is close to 1 , then he or she has has a positive contribution for the consensus to be high, while if it is close to 0 , then he or she has a negative contribution to the consensus.

Example 1: Suppose four experts $E=\left\{e_{1}, e_{2}, e_{3}, e_{4}\right\}$ which use the linguistic term set $S=\{N$ ull $(N)$, Very Low (VL), Low (L), Medium (M), High (H), Very High $(\mathrm{VH})$, Total $(\mathrm{T})\}$ to provide their linguistic preference relations on a set of four alternatives: 


$$
\begin{aligned}
P^{1} & =\left(\begin{array}{cccc}
-H & V H & L \\
L & - & T & V H \\
L & N & - & L \\
H & L & V H & -
\end{array}\right) ; P^{2}=\left(\begin{array}{cccc}
- & H & H & M \\
L & - & V H & T \\
V L & L & - & H \\
M & N & L & -
\end{array}\right) \\
P^{3} & =\left(\begin{array}{cccc}
- & H & M & V H \\
L & - & M & L \\
L & L & - & T \\
V L & H & N & -
\end{array}\right) ; P^{4}=\left(\begin{array}{cccc}
- & L & H & M \\
V H & - & M & V H \\
L & M & - & L \\
M & L & T & -
\end{array}\right)
\end{aligned}
$$

As aforementioned, to obtain the consensus degrees, we compute the different strict similarity matrix for each pair of experts using the Eq. (1):

$S M^{12}=\left(\begin{array}{cccc}- & 1.0 & 0.0 & 0.0 \\ 1.0 & - & 0.0 & 0.0 \\ 0.0 & 0.0 & - & 0.0 \\ 0.0 & 0.0 & 0.0 & -\end{array}\right) ; S M^{13}=\left(\begin{array}{ccccc}- & 1.0 & 0.0 & 0.0 \\ 1.0 & - & 0.0 & 0.0 \\ 1.0 & 0.0 & - & 0.0 \\ 0.0 & 0.0 & 0.0 & -\end{array}\right)$

$S M^{14}=\left(\begin{array}{cccc}- & 0.0 & 0.0 & 0.0 \\ 0.0 & - & 0.0 & 1.0 \\ 1.0 & 0.0 & - & 1.0 \\ 0.0 & 1.0 & 0.0 & -\end{array}\right) ; S M^{23}=\left(\begin{array}{ccccc}- & 1.0 & 0.0 & 0.0 \\ 1.0 & - & 0.0 & 0.0 \\ 0.0 & 1.0 & - & 0.0 \\ 0.0 & 0.0 & 0.0 & -\end{array}\right)$

$S M^{24}=\left(\begin{array}{cccc}- & 0.0 & 1.0 & 1.0 \\ 0.0 & - & 0.0 & 0.0 \\ 0.0 & 0.0 & - & 0.0 \\ 1.0 & 0.0 & 0.0 & -\end{array}\right) ; S M^{34}=\left(\begin{array}{ccccc}- & 0.0 & 0.0 & 0.0 \\ 0.0 & - & 1.0 & 0.0 \\ 1.0 & 0.0 & - & 0.0 \\ 0.0 & 0.0 & 0.0 & -\end{array}\right)$

Then, we compute the collective similarity matrix using the $\phi$ :

$$
S M=\left(\begin{array}{cccc}
- & 0.50 & 0.17 & 0.17 \\
0.50 & - & 0.17 & 0.17 \\
0.50 & 0.17 & - & 0.17 \\
0.17 & 0.17 & 0.00 & -
\end{array}\right)
$$

From $S M$ we obtain the following consensus degrees:

1. Consensus degrees on pairs of alternatives. The element $(i, k)$ of $S M$ represents the consensus degrees, $\operatorname{cop}_{i k}$, on the pair of alternatives $\left(x_{i}, x_{k}\right)$.

2. Consensus degrees on alternatives:

$$
c a_{1}=0.34 \quad c a_{2}=0.28 \quad c a_{3}=0.20 \quad c a_{4}=0.09
$$

3. Consensus degrees on the relation:

$$
C R=0.23
$$

Clearly, we have a low consensus degree among experts and, therefore, in a decision situation we would have to continue the negotiation process. To do so, as in [19-21] we could guide the negotiation process by means of the proximities measure. To obtain the proximity measures, we need to compute the collective fuzzy linguistic preference relation by aggregating all individual linguistic preference relations.
Using the LOWA operator [12] with the weighting vector $W=\{0.5,0.20,0.16,0.14\}$, we obtain the follow$\operatorname{ing} P^{c}$ :

$$
P^{c}=\left(\begin{array}{cccc}
- & H & H & M \\
M & - & V H & V H \\
L & L & - & H \\
M & L & H & -
\end{array}\right)
$$

The proximity matrices for each expert are:

$$
\begin{array}{r}
P M^{1}=\left(\begin{array}{cccc}
- & 1.0 & 0.0 & 0.0 \\
0.0 & - & 0.0 & 1.0 \\
1.0 & 0.0 & - & 0.0 \\
0.0 & 1.0 & 0.0 & -
\end{array}\right) ; P M^{2}=\left(\begin{array}{cccc}
- & 1.0 & 1.0 & 1.0 \\
0.0 & - & 1.0 & 0.0 \\
0.0 & 1.0 & - & 1.0 \\
1.0 & 0.0 & 0.0 & -
\end{array}\right) \\
P M^{3}=\left(\begin{array}{cccc}
- & 1.0 & 0.0 & 0.0 \\
0.0 & - & 0.0 & 0.0 \\
1.0 & 1.0 & - & 0.0 \\
0.0 & 0.0 & 0.0 & -
\end{array}\right) ; P M^{4}=\left(\begin{array}{cccc}
- & 0.0 & 1.0 & 1.0 \\
0.0 & - & 0.0 & 1.0 \\
1.0 & 0.0 & - & 0.0 \\
1.0 & 1.0 & 0.0 & -
\end{array}\right)
\end{array}
$$

And then, the proximity measures are:

1. Proximity measure on pairs of alternatives. The proximity measure of an expert $e_{h}$ on a pair of alternatives $\left(x_{i}, x_{k}\right)$ to the group's one, $p p_{i k}^{h}$, is expressed by the element $(i, k)$ of the proximity matrix $P M^{h}$.

2. Proximity measure on alternatives:

$$
\begin{aligned}
& \left\{p a_{1}^{1}, p a_{2}^{1}, p a_{3}^{1}, p a_{4}^{1}\right\}=\{0.33,0.50,0.17,0.33\} \\
& \left\{p a_{1}^{2}, p a_{2}^{2}, p a_{3}^{2}, p a_{4}^{2}\right\}=\{0.67,0.50,0.67,0.50\} \\
& \left\{p a_{1}^{3}, p a_{2}^{3}, p a_{3}^{3}, p a_{4}^{3}\right\}=\{0.33,0.33,0.33,0.00\} \\
& \left\{p a_{1}^{4}, p a_{2}^{4}, p a_{3}^{4}, p a_{4}^{4}\right\}=\{0.67,0.33,0.33,0.67\}
\end{aligned}
$$

3. Proximity measure on the relation:

$$
p r^{1}=0.33 \quad p r^{2}=0.58 \quad p r^{3}=0.25 \quad p r^{4}=0.50
$$

With these scores the experts 1 and 3 should change highly their positions to increase the level of consensus in the next consensus rounds.

\subsection{Consensus models based on soft coincidence among preferences}

As above, similarity criteria among preferences are used to compute the coincidence concept. However, in this case, a major number of possible coincidence degrees is considered. It is assumed that the coincidence concept is a gradual concept which could be assessed with different degrees defined in the unit interval $[0,1]$. These are the more popular consensus models. Some examples of this approach are the following:

- In [25], a first consensus model based on soft coincidence was also defined. But in this case, given a particular alternative pair and two experts, the coincidence among their preference is measured using a closeness function $s:[0,1] \rightarrow[0,1]$. 
- In [26,27], some soft consensus measures are introduced and defined as extensions of those presented in [25], considering GDM problems with heterogeneous set of alternatives and heterogeneous groups of experts, respectively.

- An extension of these models is presented in [10], which consists in the computation of consensus measures using the ordered weighted averaging (OWA) operator [37].

- In [5], a soft consensus model for multi-criteria GDM problems defined in a ordinal fuzzy linguistic approach was defined. In this case, coincidence values are obtained by means of a linguistic similarity function defined directly on linguistic assessments given on the alternatives.

- In [13], the fuzzification of soft coincidence concept was presented. The soft coincidence is defined in each alternative pair of a linguistic preference relation as a fuzzy set defined on the set of expert pairs and characterized by closeness observed among their preferences. The closeness among preferences is established by means of ad-hoc closeness table defined among all the possible labels of linguistic term set used to represent the preferences.

- In [20], a soft consensus approach is presented to deal with GDM problems in a multi-granular fuzzy linguistic context. Three kinds of soft consensus measures are considered as in $[11,13,14]$. In this case, the soft coincidence among multi-granular linguistic preferences is obtained using a similarity function defined on transformation of such preferences in a basic linguistic term set.

- In [21], a soft consensus model based on three consensus measures was also proposed. In this case, experts provide their preferences by means of incomplete fuzzy preference relations assessed in $[0,1]$ and the soft coincidence is defined using a similarity function among preferences in $[0,1]$.

- Finally, in [6], a soft consensus model is presented for GDM problems in a unbalanced fuzzy linguistic context $[17,24]$. In this case, as in $[11,13,14]$, the soft coincidence is computed using a similarity function defined on transformation of unbalanced fuzzy linguistic preferences in a basic linguistic term set.

Following in the framework used previously, we could apply a consensus model based on soft coincidence in a fuzzy GDM problem based on linguistic preference relations as follows:

1. Compute the similarity matrices $S M^{h l}=\left[s m_{i k}^{h l}\right]$, $i, k=1, \ldots, n$, :

$$
s m_{i k}^{h l}=s\left(p_{i k}^{h}, p_{i k}^{l}\right)
$$

where $s\left(p_{i k}^{h}, p_{i k}^{l}\right)$ is a similarity function which measures the coincidence between the opinions $p_{i k}^{h}$ and $p_{i k}^{l}$. Depending on the fuzzy context, different similarity functions can be used [20,21].

2. Then, a collective similarity matrix, $S M=\left[s m_{i k}\right]$, is obtained by aggregating all the similarity matrices using the arithmetic mean $\phi$ :

$s m_{i k}=\phi\left(s m_{i k}^{h l}, h=1, \ldots, m-1, l=h+1, \ldots, m\right)$

3. Compute the consensus degrees and proximity measures:

(a) Consensus Degrees: Once the similarity matrices are computed, the consensus degrees are calculated at three different levels as in the consensus models based on strict coincidence among preferences:

i. Level 1. Consensus degree on pairs of alternatives:

$\operatorname{cop}_{i k}=s m_{i k}$

ii. Level 2. Consensus degree on alternatives:

$$
c a_{i}=\frac{\sum_{k=1 ; k \neq i}^{n}\left(\operatorname{cop}_{i k}+\operatorname{cop}_{k i}\right)}{2 n-2}
$$

iii. Level 3. Consensus degree on the relation:

$$
C R=\frac{\sum_{i=1}^{n} c a_{i}}{n}
$$

(b) Proximity Measures: To compute the proximity measures for each expert, we need to obtain the collective preference relation, $P^{c}=\left[p_{i k}^{c}\right]$, which is computed as follows:

$p_{i k}^{c}=\phi\left(p_{i k}^{1}, \ldots, p_{i k}^{m}\right)$

To do so, the LOWA operator [12] can be used. Using $P^{c}$, for each expert, $e_{h}$, a proximity matrix, $P M^{h}=\left[p m_{i k}^{h}\right]$, is obtained:

$p m_{i k}^{h l}=s\left(p_{i k}^{h}, p_{i k}^{c}\right)$

Finally, the computation of the proximity measures is carried out at three different level as follows:

i. Level 1. Proximity measure on pairs of alternatives:

$$
p p_{i k}^{h}=p m_{i k}^{h}
$$

ii. Level 2. Proximity measure on alternatives:

$$
p a_{i}^{h}=\frac{\sum_{k=1, k \neq i}^{n}\left(p p_{i k}^{h}+p p_{k i}^{h}\right)}{2 n-2}
$$


iii. Level 3. Proximity measure on the relation:

$$
p r^{h}=\frac{\sum_{i=1}^{n} p a_{i}^{h}}{n}
$$

Example 2: Assuming the same linguistic preference relations provided by the experts in the above example, the soft consensus degrees are obtained as follows.

To obtain the consensus degrees, firstly, we compute the different similarity matrix for each pair of experts. In this case, we need to define a similarity function. As we assume a fuzzy linguistic framework, the following similarity function can be used:

$s\left(s_{i}, s_{j}\right)=1-\frac{|i-j|}{g}$

Using this similarity function, the following similarity matrices are obtained:

$S M^{12}=\left(\begin{array}{cccc}- & 1.00 & 0.83 & 0.83 \\ 1.00 & - & 0.83 & 0.83 \\ 0.83 & 0.67 & - & 0.67 \\ 0.83 & 0.67 & 0.50 & -\end{array}\right) ; S M^{13}=\left(\begin{array}{cccc}- & 1.00 & 0.67 & 0.50 \\ 1.00 & - & 0.50 & 0.50 \\ 1.00 & 0.67 & - & 0.33 \\ 0.50 & 0.67 & 0.17 & -\end{array}\right)$

$S M^{14}=\left(\begin{array}{cccc}- & 0.67 & 0.83 & 0.83 \\ 0.50 & - & 0.50 & 1.00 \\ 1.00 & 0.50 & - & 1.00 \\ 0.83 & 1.00 & 0.83 & -\end{array}\right) ; S M^{23}=\left(\begin{array}{cccc}- & 1.00 & 0.83 & 0.67 \\ 1.00 & - & 0.67 & 0.33 \\ 0.83 & 1.00 & - & 0.67 \\ 0.67 & 0.33 & 0.67 & -\end{array}\right)$

$S M^{24}=\left(\begin{array}{cccc}- & 0.50 & 1.00 & 1.00 \\ 0.33 & - & 0.67 & 0.83 \\ 0.83 & 0.83 & - & 0.67 \\ 1.00 & 0.67 & 0.33 & -\end{array}\right) ; S M^{34}=\left(\begin{array}{cccc}- & 0.67 & 0.83 & 0.67 \\ 0.50 & - & 1.00 & 0.50 \\ 1.00 & 0.83 & - & 0.33 \\ 0.67 & 0.67 & 0.00 & -\end{array}\right)$

Then, we compute the collective similarity matrix:

$$
S M=\left(\begin{array}{cccc}
- & 0.81 & 0.83 & 0.75 \\
0.72 & - & 0.62 & 0.66 \\
0.92 & 0.75 & - & 0.27 \\
0.75 & 0.67 & 0.42 & -
\end{array}\right)
$$

Finally, we obtain the following consensus degrees:

1. Consensus degrees on pairs of alternatives. The element $(i, k)$ of $s m$ represents the consensus degrees on the pair of alternatives $\left(x_{i}, x_{k}\right)$.

2. Consensus degrees on alternatives:

$$
c a_{1}=0.80 \quad c a_{2}=0.71 \quad c a_{3}=0.63 \quad c a_{4}=0.59
$$

3. Consensus degrees on the relation:

$$
C R=0.68
$$

According to this score, we can affirm that the consensus level is acceptable in contrast to the Example (1) based on the strict coincidence.

Proximity measures are obtained from the collective fuzzy linguistic preference relation, which using the LOWA operator with the weighting vector $W=$ $\{0.5,0.20,0.16,0.14\}$, is the following:

$$
P^{c}=\left(\begin{array}{cccc}
- & H & H & M \\
M & - & V H & V H \\
L & L & - & H \\
M & L & H & -
\end{array}\right)
$$

From $P^{c}$, the proximity matrices for each expert are:

$P M^{1}=\left(\begin{array}{cccc}- & 1.00 & 0.83 & 0.83 \\ 0.83 & - & 0.83 & 1.00 \\ 1.00 & 0.67 & - & 0.67 \\ 0.83 & 1.00 & 0.83 & -\end{array}\right) ; P M^{2}=\left(\begin{array}{cccc}- & 1.00 & 1.00 & 1.00 \\ 0.83 & - & 1.00 & 0.83 \\ 0.83 & 1.00 & - & 1.00 \\ 1.00 & 0.67 & 0.67 & -\end{array}\right)$

$P M^{3}=\left(\begin{array}{cccc}- & 1.00 & 0.83 & 0.67 \\ 0.83 & - & 0.67 & 0.50 \\ 1.00 & 1.00 & - & 0.67 \\ 0.67 & 0.67 & 0.33 & -\end{array}\right) ; P M^{4}=\left(\begin{array}{cccc}- & 0.67 & 1.00 & 1.00 \\ 0.67 & - & 0.67 & 1.00 \\ 1.00 & 0.83 & - & 0.67 \\ 1.00 & 1.00 & 0.67 & -\end{array}\right)$

1. Proximity measure on pairs of alternatives. The proximity measure of an expert $e_{h}$ on a pair of alternatives $\left(x_{i}, x_{k}\right)$ to the group's one, $p p_{i k}^{h}$, is expressed by the element $(i, k)$ of the proximity matrix $P M^{h}$.

2. Proximity measure on alternatives:

$$
\begin{aligned}
& \left\{p a_{1}^{1}, p a_{2}^{1}, p a_{3}^{1}, p a_{4}^{1}\right\}=\{0.89,0.89,0.80,0.86\} \\
& \left\{p a_{1}^{2}, p a_{2}^{2}, p a_{3}^{2}, p a_{4}^{2}\right\}=\{0.94,0.89,0.92,0.86\} \\
& \left\{p a_{1}^{3}, p a_{2}^{3}, p a_{3}^{3}, p a_{4}^{3}\right\}=\{0.83,0.79,0.75,0.58\} \\
& \left\{p a_{1}^{4}, p a_{2}^{4}, p a_{3}^{4}, p a_{4}^{4}\right\}=\{0.89,0.81,0.81,0.89\}
\end{aligned}
$$

3. Proximity measure on the relation:

$$
p r^{1}=0.86 \quad p r^{2}=0.90 \quad p r^{3}=0.74 \quad p r^{4}=0.85
$$

In this case, unlike Example (1) all experts present adequate proximity measures, and the experts with worse scores are $e_{3}$ and $e_{4}$.

2.3 Consensus models based on coincidence among on solutions

In this case, similarity criteria among the solutions obtained from the experts' preferences are used to compute the coincidence concept and different degrees assessed in $[0,1]$ are assumed $[2,19]$. Basically, we compare the positions of the alternatives between the individual solutions and the collective solution, which allows to know better the real consensus situation in each moment of the consensus process. Some examples of this approach are the following:

- In [19] was defined the first consensus model based on the measuring the coincidence degree between individual solutions and collective solution. It is assumed that experts represent their preferences by 
means of different elements of representation (relation, ordering and utilities) and then it is not possible to compare preferences. To overcome this problem, authors propose to compare solutions to obtain the coincidence degrees. This means that the first step of consensus process to measure coincidence degrees is to apply a selection process to obtain a temporary collective solution and the temporary individual solutions, and measure the closeness among them. An important characteristic of this consensus model was the introduction of a recommendation system to aid experts to change their preferences in the consensus reaching process and, in such a way, to substitute the moderator's actions.

- In [2], a similar consensus model is presented but assuming heterogeneous GDM problems, i.e., experts with different importance degrees.

Following the consensus model defined in [19], which only is based on consensus degrees not proximity measures, we can define a consensus model based on coincidence among solutions for fuzzy GDM problems with linguistic preference relations as follows:

1. To obtain the collective ordered vector of alternatives (temporary collective solution) $V^{c}$. To do so, we apply a selection process in two steps the selection process $[1,7,34]$ :

(a) Aggregation. In this step, a collective preference relation $P^{c}=\left(p_{i k}^{c}\right)$ is obtained by means of the aggregation of all individual preference relations $\left\{P^{1}, P^{2}, \ldots, P^{m}\right\}$. This collective relation indicates the global preference between every ordered pair of alternatives according to the majority of experts' opinions.

(b) Exploitation. In this step, the set of solution alternatives is obtained from the collective preference relation. In this consensus model we call it as collective ordered vector of alternatives. To do so, different choice degrees of alternatives could be used $[16,23]$.

2. Calculating the individual ordered vector of alternatives (individual solution) $V^{h}$ for every expert $e_{h}$. To do it, we apply directly the exploitation step on each individual linguistic preference relation $P^{h}$.

3. Calculating the proximity of each expert $e_{h}$ for each alternative $x_{i}$, called $p^{h}\left(x_{i}\right)$, by comparing the ranking positions of that alternative in the experts' individual solution $V^{h}$ (symbolized by $V_{i}^{h}$ ) and in the collective solution $V^{c}$ (symbolized by $V_{i}^{c}$ ) as $p^{h}\left(x_{i}\right)=p\left(V^{h}, V^{c}\right)\left(x_{i}\right)=f\left(\left|V_{i}^{c}-V_{i}^{h}\right|\right)$. As a general dissimilarity function, $f(x)=(a \cdot x)^{b}, 1 \geq b \geq 0$ may be considered, and, in particular, the function taking $a=1 /(n-1)$ may be used, and then

$$
\begin{aligned}
& p^{h}\left(x_{i}\right)=p\left(V^{h}, V^{c}\right)\left(x_{i}\right)=f\left(\left|V_{i}^{c}-V_{i}^{h}\right|\right)= \\
& =\left(\frac{\left|V_{j}^{c}-V_{i}^{h}\right|}{n-1}\right)^{b} \in[0,1]
\end{aligned}
$$

The parameter $b$ controls the rigorousness of the consensus process, in such a way, that values of $b$ close to one decrease the rigorousness and, therefore, the number of rounds to develop in the group discussion process, and values of $b$ close to zero increase the rigorousness and, therefore, the number of rounds. Appropriate values for $b$ are: $0.5,0.7,0.9$, 1.

4. Calculating the consensus degree of all experts on each alternative $x_{i}$ using the following expression:

$C\left(x_{i}\right)=1-\sum_{h=1}^{m} \frac{p^{h}\left(x_{i}\right)}{m}$

5. The consensus measure over the set of alternatives, called $C_{X}$, will be calculated by the aggregation of the above consensus degrees on the alternatives. It is considered that the consensus degrees about the solution set of alternatives has to take a more important weight in this aggregation. To do so, in [19] it was used the S-OWA OR-LIKE operator defined by Yager and Filev [38]:

$$
\begin{aligned}
& C_{X}=S_{O W A O R-L I K E}\left(\left\{C\left(x_{s}\right) ; x_{s} \in X_{\text {sol }}\right\},\right. \\
& \left.\left\{C\left(x_{t}\right) ; x_{t} \in X-X_{s o l}\right\}\right)= \\
& =(1-\beta) \cdot \sum_{t=1}^{\nu} \frac{C\left(x_{t}\right)}{\nu}+\beta \cdot \sum_{s=1}^{\gamma} \frac{C\left(x_{s}\right)}{\gamma}
\end{aligned}
$$

where $\gamma$ is the cardinal of the set $X_{\text {sol }} ; \nu$ is the cardinal of the set $X-X_{\text {sol }} ; \beta \in[0,1]$. $\beta$ is a parameter to control the OR-LIKE behavior of the aggregation operator. The higher the value of $\beta$, the higher the influence of the consensus degrees of the solution alternatives on the global consensus degree.

Example 3: Assuming the same linguistic preference relations provided by the experts in the above examples, the soft consensus degrees based on coincidence among solutions are obtained as follows:

1. Obtaining the collective ordered vector of alternatives $V^{c}$ :

(a) Aggregation: Using the LOWA operator [12] and the weighting vector $W=\{0.5,0.20,0.16,0.14\}$, the following collective linguistic preference relation is obtained:

$$
P^{c}=\left(\begin{array}{cccc}
- & H & H & M \\
M & - & V H & V H \\
L & L & - & H \\
M & L & H & -
\end{array}\right)
$$


(b) Exploitation: We use a choice degree called dominance degree [16] to characterize the alternatives and compute the ordered vector of alternatives:

$$
D D_{i}=\phi\left(p_{i 1}^{c}, p_{i 2}^{c}, \ldots, p_{i(i-1)}^{c}, p_{i(i+1)}^{c}, \ldots, p_{i n}^{c}\right)
$$

To do so, we use the LOWA operator with the weighting vector $W=\{0.54,0.28,0.18\}$. Then the dominance degrees $\left\{D D_{1}, \ldots, D D_{4}\right\}$ are the following:

$$
\begin{array}{ll}
D D_{1}=M, & D D_{2}=H \\
D D_{3}=M, & D D_{4}=M
\end{array}
$$

And thus, the collective ordered vector of alternatives is $\left\{x_{2}, x_{1}, x_{3}, x_{4}\right\}$.

2. Calculating $\left\{V^{h} ; h=1, \ldots, m\right\}$ :

$$
\begin{array}{ll}
e_{1}:\left\{x_{2}, x_{1}, x_{4}, x_{3}\right\}, & e_{2}:\left\{x_{2}, x_{1}, x_{3}, x_{4}\right\} \\
e_{3}:\left\{x_{1}, x_{3}, x_{2}, x_{4}\right\}, & e_{4}:\left\{x_{2}, x_{4}, x_{1}, x_{3}\right\}
\end{array}
$$

3. The differences between the ranking of alternatives in the temporary collective solution and the individual solution are:

\begin{tabular}{|c|c|c|c|c|}
\hline$V_{i}^{c}-V_{i}^{h}$ & $x_{1}$ & $x_{2}$ & $x_{3}$ & $x_{4}$ \\
\hline$e_{1}$ & 0 & 0 & -1 & 1 \\
\hline$e_{2}$ & 0 & 0 & 0 & 0 \\
\hline$e_{3}$ & 1 & 1 & -2 & 0 \\
\hline$e_{4}$ & 0 & 2 & -1 & -1 \\
\hline
\end{tabular}

4. Consensus degrees on alternatives calculated for $b=$ 1:

$$
\left(C\left(x_{1}\right), C\left(x_{2}\right), C\left(x_{3}\right), C\left(x_{4}\right)\right)=(0.83,1.0,0.67,0.67)
$$

5. Consensus measure calculated for $b=1$ and $\beta=0.6$ is:

$$
C_{X}=0.88
$$

As we observe, assuming the same framework considered in Examples (1) and (2), we obtain a higher consensus level with this consensus model, which reflects better the actual decision situation.

\section{Discussion}

In this section, we analyze the advantages and drawbacks of the different fuzzy soft consensus approaches.
1. Strict coincidence among preferences. This consensus approach assumes only two possible values: 1 if the opinions are equal and, otherwise, a value of 0 . Therefore, as we have seen in the Example (1), the advantage of this approach is that the computation of the consensus degrees is simple and easy. However, the drawback of this approach is that the consensus degrees obtained do not reflect the real consensus situation because it only assigns values of 1 or 0 when comparing the experts' opinions, and, for example, we obtain a consensus value 0 for two different preference situations as (very high, high) and (very high,low), when clearly in the second case the consensus value should be lower than in the first case. It can be seen in the Example (1), where the degree of consensus obtained is very low (0.23) although checking the preference relations provided by the experts, we can observe that the consensus among the experts is higher.

2. Soft coincidence among preferences. In this approach, similarity criteria among preferences are used to compute the coincidence concept but, in this case, it is assumed that the coincidence concept is a gradual concept which could be assessed with different degrees defined in $[0,1]$. The advantage of this approach is that the consensus degrees obtained reflect better the real consensus situation. Comparing Examples (1) and (2), this is clearly observed. However, the drawback of this approach is that the computation of the consensus degrees is more difficult because we need to define similarity criteria to compute the consensus measures, and, sometimes, as it happens in $[6,20]$, it is not possible to define these similarity measures directly.

3. Coincidence among solutions. The advantage of this approach is that the consensus degrees are obtained comparing not the opinions but the position of the alternatives in each solution, what allows us to reflect the real consensus situation in each moment of the consensus reaching process, as it happens in the Example (3). However, the drawback of this approach is that the computation of the consensus degrees is more difficult than in the above approaches because we need to define similarity criteria and it is necessary to apply a selection process before obtaining the consensus degrees. As we show in Example (3) the computation of the consensus degrees is more complex.

\section{Advanced consensus approaches}

In this section, we describe the soft advanced consensus approaches which have been developed using the above 
concepts of coincidence. These consensus approaches are mainly two: ones generate recommendations to help experts and others develop adaptive consensus processes. We present them in the following subsections in depth.

\subsection{Consensus approaches generating} recommendations to help experts

These approaches generate simple and easy rules to help experts change their opinions and find out which direction that change should follow in order to obtain the highest degree of consensus possible [19,21].

To do so, they are based on two consensus criteria, consensus degrees indicating the agreement between experts opinions and proximity measures used to find out how far the individual opinions are from the group opinion. Thus, proximity measures are used in conjunction with the consensus degrees to build a guidance advice system, which acts as a feedback mechanism that generates recommendations so that experts can change their opinions. Furthermore, these consensus criteria are computed at the three different levels of representation of information of a preference relation: pair of alternatives, alternative, and relation. In such a way, we will be able to identify which experts are close to the consensus solution, or in which alternatives the experts are having more trouble to reach consensus.

So, the computation of the consensus degrees in this advanced consensus approaches is carried out using the Eq. (3)-(5), i.e., as in the above consensus models. Once consensus degrees are calculated, the proximity measures are obtained. To compute them for each expert, the Eq. (9)-(11) are used.

As aforementioned, if the proximity measures are close to 1 , then they have a positive contribution for the consensus to be high, while if they are close to 0 , then they have a negative contribution to the consensus. Therefore, we can use them to provide advice to the experts to change their opinions and to find out which direction that change has to follow in order to obtain the highest degree of consensus possible.

Thus, once proximity measures are calculated, the recommendations to help experts change their opinions are generated. The production of advice to achieve a solution with the highest degree of consensus possible is carried out using two kinds of rules [20]: Identification rules and Direction rules.

1. Identification rules (IR). We must identify the experts, alternatives and pairs of alternatives contributing less to reach a high degree of consensus and, therefore, should participate in the change process. (a) Identification rule of experts (IR.1). It identifies the set of experts that should receive advice on how to change some of their preference values. This set of experts, called EXPCH, that should change their opinions are those whose satisfaction degree on the relation is lower than the minimum consensus threshold $\gamma$. Therefore, the identification rule of experts, IR.1, is the following:

$$
E X P C H=\left\{e_{h} \mid p r^{h}<\gamma\right\}
$$

(b) Identification rule of alternatives (IR.2). It identifies the alternatives whose associated assessments should be taken into account by the above experts in the change process of their preferences. This set of alternatives is denoted as $A L T$. The identification rule of alternatives, IR.2, is the following:

$$
A L T=\left\{x_{i} \in X \mid p a_{i}^{h}<\gamma \wedge e_{h} \in E X P C H\right\}
$$

(c) Identification rule of pairs of alternatives (IR.3). It identifies the particular pairs of alternatives $\left(x_{i}, x_{k}\right)$ whose respective associated assessments $p_{i k}^{h}$ the expert $e_{h}$ should change. This set of pairs of alternatives is denoted as $P A L T^{h}$. The identification rule of pairs of alternatives, IR.3, is the following:

$$
\begin{aligned}
& P A L T^{h}=\left\{\left(x_{i}, x_{k}\right) \mid x_{i} \in A L T \wedge\right. \\
& \left.\wedge e_{h} \in E X P C H \wedge p p_{i k}^{h}<\gamma\right\}
\end{aligned}
$$

2. Direction rules (DR). We must find out the direction of the change to be recommended in each case, i.e., the direction of change to be applied to the preference assessment $p_{i k}^{h}$, with $\left(x_{i}, x_{k}\right) \in P A L T^{h}$. To do this, we define the following two direction rules.

(a) DR.1. If $p_{i k}^{h}>p_{i k}^{c}$, the expert $e_{h}$ should decrease the assessment associated to the pair of alternatives $\left(x_{i}, x_{k}\right)$, i.e., $p_{i k}^{h}$.

(b) DR.2. If $p_{i k}^{h}<p_{i k}^{c}$, the expert $e_{h}$ should increase the assessment associated to the pair of alternatives $\left(x_{i}, x_{k}\right)$, i.e., $p_{i k}^{h}$.

\subsection{Adaptive consensus approaches}

These consensus approaches are based on a refinement process of the consensus process that allows to increase the agreement and to reduce the number of experts' preferences that should be changed after each consensus round [31]. The refinement process adapts the search for 


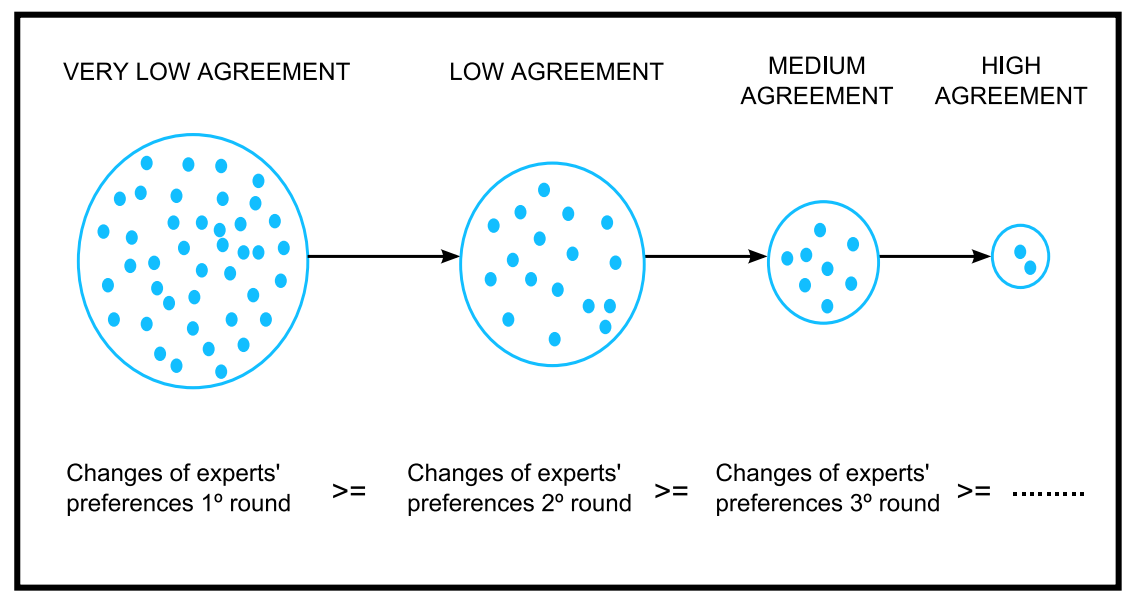

Fig. 2 Reduction of the number of changes of preferences into the consensus process

the furthest experts' preferences to the existent agreement in each round of consensus. So, when the agreement is very low (initial rounds of the consensus process), the number of changes of preferences should be bigger than when the agreement is medium or high (final rounds) (see Fig. 2).

These approaches consider that in the first rounds of the consensus process, the agreement is usually very low and it seems logic that many experts' preferences should be changed. However, after several rounds, the agreement should have improved and then just the furthest experts' preferences from the collective preference should be changed. It involves that the procedure to search for the furthest experts' preferences from collective preference should be different according to the achieved agreement in each round. Each Preference Search Procedure (PSp) should have a different behavior and should return a different set of preferences that each expert should change in order to improve the agreement in the next consensus round. In consequence of the adaptation of the consensus process to the existent agreement in each round, the number of changes of preferences suggested to experts after each consensus round will be smaller according to the favorable evolution of the level of agreement.

In such a way, in the consensus process, if the agreement among experts is low, i.e, there are a lot of experts' preferences with different assessments, the number of experts which should change their preferences in order to make them closer to collective preference should be great. However, if the agreement is medium or high, it means that the majority of preferences are similar and therefore there exist a low number of experts' preferences far from the collective preference. In this case, only these experts should change them in order to improve the agreement. Keeping in mind this idea, these approaches propose distinguishing among three level of agreement: very low, low and medium consensus. Each level of consensus involves to carry out the search for the furthest preferences in a different way. So when the consensus degree $C R$ is very low, these approaches will search for the furthest preferences on all experts, while if $C R$ is medium, the search will be limited to the furthest experts. To do so, these approaches carries out three different PSps:

- PSp for very low consensus,

- PSp for low consensus, and

- PSp for medium consensus.

The possibility of carrying out different PSps according to the existent consensus degree in each round defines the adaptive character of the model.

\section{Concluding remarks}

We have analyzed different consensus approaches to compute soft consensus measures in fuzzy GDM problems. Additionally, we have described the new advanced approaches, i.e., those approaches allowing to generate recommendations to help experts change their opinions in order to obtain the highest degree of consensus possible, and, on the other hand, those approaches adapting the consensus process to increase the agreement and reduce the number of experts' preferences that should be changed after each consensus round.

Acknowledgements This paper has been developed with the financing of SAINFOWEB project (TIC00602), Feder Funds in FUZZYLING project (TIN2007-61079) and PETRI project (PET 2007-0460). 


\section{References}

1. Alonso S, Cabrerizo FJ, Chiclana F, Herrera F, HerreraViedma E (2009) Group decision-making with incomplete fuzzy linguistic preference relations. International Journal of Intelligent Systems 24:201-222

2. Ben-Arieh D, Chen Z (2006) Linguistic labels aggregation and consensus measure for autocratic decision-making using group recommendations. IEEE Transactions on Systems, Man, and Cybernetics, Part A: Systems and Humans 36:558-568

3. Bezdek J, Spillman B, Spillman R (1977) Fuzzy measures of preferences and consensus in group decision making. In: Proceedings of 1977 IEEE conference on decision and control, pp 1303-1309

4. Bezdek J, Spillman B, Spillman R (1978) A fuzzy relation space for group decision theory. Fuzzy Sets and Systems 1:255268

5. Bordogna G, Fedrizzi M, Pasi G (1997) A linguistic modeling of consensus for a fuzzy majority in group decision making. IEEE Transactions on Systems, Man and Cybernetics 27:126132

6. Cabrerizo FJ, Alonso S, Herrera-Viedma E (2009) A consensus model for group decision making problems with unbalanced fuzzy linguistic information. International Journal of Information Technology \& Decision Making 8:1-23

7. Chiclana F, Herrera F, Herrera-Viedma E (1998) Integrating three representation models in fuzzy multipurpose decision making based on fuzzy preference relations. Fuzzy Sets and Systems 97:33-48

8. Chen SJ, Hwang CL (1992) Fuzzy multiple attributive decision making: Theory and its applications. Springer, Berlin

9. Delgado M, Verdegay JL, Vila MA (1993) On aggregation operations of linguistic labels. International Journal of Intelligent Systems 8:351-370

10. Fedrizzi M, Kacprzyk J, Nurmi H (1993) Consensus degrees under fuzzy majorities and fuzzy preferences using OWA (ordered weighted average) operators. Control Cybernet 22:78-86

11. Herrera F, Herrera-Viedma E, Verdegay JL (1996) A model of consensus in group decision making under linguistic assessments. Fuzzy Sets and Systems 78:73-87

12. Herrera F, Herrera-Viedma E, Verdegay JL (1996) Direct approach processes in group decision making using linguistic OWA operators. Fuzzy Sets and Systems 79:175-190

13. Herrera F, Herrera-Viedma E, Verdegay JL (1997) Linguistic measures based on fuzzy coincidence for reaching consensus in group decision making. International Journal of Approximate Reasoning 16:309-334

14. Herrera F, Herrera-Viedma E, Verdegay JL (1997) A rational consensus model in group decision making using linguistic assessments. Fuzzy Sets and Systems 88:31-49

15. Herrera F, Herrera-Viedma E, Verdegay JL (1998) Choice processes for non-homogeneous group decision making in linguistic setting. Fuzzy Sets and Systems 94:287-308

16. Herrera F, Herrera-Viedma E (2000) Choice functions and mechanisms for linguistic preference relations. European Journal of Operational Research 120:144-161

17. Herrera F, Herrera-Viedma E, Martínez M (2008) A fuzzy linguistic methodology to deal with unbalanced linguistic term sets. IEEE Transactions on Fuzzy Systems 16:354-370

18. Herrera-Viedma E (2001) An information retrieval system with ordinal linguistic weighted queries based on two weighting elements. Int. J. of Uncertainty, Fuzziness and KnowledgeBased Systems, 9: 77-88

19. Herrera-Viedma E, Herrera F, Chiclana F (2002) A consensus model for multiperson decision making with different preference structures. IEEE Transactions on Systems, Man and Cybernetics, Part A: Systems and Humans 32:394-402
20. Herrera-Viedma E, Martínez L, Mata F, Chiclana F (2005) A consensus support system model for group decision-making problems with multi-granular linguistic preference relations. IEEE Transaction on Fuzzy Systems 13:644-658

21. Herrera-Viedma E, Alonso S, Chiclana F, Herrera F (2007) A consensus model for group decision making with incomplete fuzzy preference relations. IEEE Transactions on Fuzzy Systems 15:863-877

22. Herrera-Viedma E, Pasi G, López-Herrera AG, Porcel C (2006) Evaluating the information quality of web sites: A methodology based on fuzzy computing with words. J. of the American Society for Information Science and Technology 57(4):538-549

23. Herrera-Viedma E, Chiclana F, Herrera F, Alonso S (2007) A group decision-making model with incomplete fuzzy preference relations based on additive consistency. IEEE Transactions on Systems, Man and Cybernetics, Part B: Cybernetics $37: 176-189$

24. Herrera-Viedma E, López-Herrera AG (2007) A model of information retrieval system with unbalanced fuzzy linguistic information. International Journal of Intelligent Systems $22: 1197-1214$

25. Kacprzyk J (1987) On some fuzzy cores and soft consensus measures in group decision making. In: Bezdek J (ed) The Analysis of Fuzzy Information, CRC Press, pp. 119-130

26. Kacprzyk J, Fedrizzi M (1986) Soft consensus measure for monitoring real consensus reaching processes under fuzzy preferences. Control Cybernet 15:309-323

27. Kacprzyk J, Fedrizzi M (1988) A soft measure of consensus in the setting of partial (fuzzy) preferences. European Journal of Operational Research 34:316-323

28. Kacprzyk J, Fedrizzi M, Nurmi H (1992) Group decision making and consensus under fuzzy preferences and fuzzy majority. Fuzzy Sets and Systems 49:21-31

29. Kacprzyk J, Nurmi H, Fedrizzi M (1997) Consensus under fuzziness. Kluwer Academic Publishers, Boston

30. Lu J, Zhang G, Ruan D (2008) Intelligent multi-criteria fuzzy group decision-making for situation assessments. Soft Computing 12:289-299

31. Mata F, Martínez L, Herrera-Viedma E (2009) An adaptive consensus support model for group decision making problems in a multi-granular fuzzy linguistic context. IEEE Transactions on Fuzzy Systems 17:2 279-290

32. Montero J (2008) The impact of fuzziness in social choice paradoxes. Soft Computing 12:177-182

33. Nurmi H (2008) Fuzzy social choice: a selective retrospect. Soft Computing 12:281-288

34. Roubens M (1997) Fuzzy sets and decision analysis. Fuzzy Sets and Systems 90:199-206

35. Spillman B, Bezdek J, Spillman R (1979) Coalition analysis with fuzzy sets. Kybernetes 8:203-211.

36. Spillman B, Spillman R, Bezdek J (1980) A fuzzy analysis of consensus in small groups. In Wang PP, Chang SK (eds) Fuzzy Automata and Decision Processes, North-Holland, pp 331-356

37. Yager RR (1988) On ordered weighted averaging aggregation operators in multicriteria decision making. IEEE Transactions on Systems, Man, Cybernetics, Part A: Systems and Humans 18:183-190

38. Yager RR, Filev DP (1994) Parameterized and-like and orlike OWA operators. International Journal of General Systems 22:297-316

39. Zadeh LA (1983) A computational approach to fuzzy quantifiers in natural languages. Computers and Mathematics with Applications 9:149-184 